\title{
Approximation Properties of Kantorovich Type Modifications of $(p, q)$-Meyer-König-Zeller Operators
}

\author{
HONEy SHARMA, RAMAPATI MAURYA*, AND CHEENA GUPTA
}

ABSTRACT. In this paper, we introduce Kantorovich type modification of $(p, q)$-Meyer-König-Zeller operators. We estimate rate of convergence of proposed operators using modulus of continuity and Lipschitz class functions. Further, we obtain the statistical convergence and local approximation results for these operators. In the last section, we estimate the rate of convergence of $(p, q)$-Meyer-König-Zeller Kantorovich operators by means of Matlab programming.

Keywords: $(p, q)$-Calculus, $(p, q)$-Meyer-König-Zeller operators, Modulus of continuity, Statistical Convergence, Peetre's K-functional.

2010 Mathematics Subject Classification: 41A25, 41A35.

\section{INTRODUCTION}

In 1960, Meyer-König and Zeller [22] defined the operators known as Meyer-König-Zeller (MKZ) operators, as follows:

$$
\begin{aligned}
& M_{n}(f ; x)=\sum_{k=0}^{\infty} f\left(\frac{k}{n+k}\right)\left(\begin{array}{c}
n+k \\
k
\end{array}\right) x^{k}(1-x)^{n+1}, \text { if } x \in[0,1), \\
& M_{n}(f ; 1)=f(1) \text {, if } x=1, n \in \mathbb{N} .
\end{aligned}
$$

Further, Cheney and Sharma [3] modified these operators and introduced a new form of the Meyer-König-Zeller operators, as follows:

$$
\begin{aligned}
& M_{n}(f ; x)=\sum_{k=0}^{\infty} f\left(\frac{k}{n+k+1}\right)\left(\begin{array}{c}
n+k \\
k
\end{array}\right) x^{k}(1-x)^{n+1}, \text { if } x \in[0,1), \\
& M_{n}(f ; 1)=f(1), \text { if } x=1, n \in \mathbb{N} .
\end{aligned}
$$

In 2000, T. Trif [21] introduced the $q$-Meyer-König-Zeller operators for $f \in C[0,1]$, as follows:

$$
\begin{aligned}
& M_{n, q}(f ; x)=\sum_{k=0}^{\infty} f\left(\frac{[k]_{q}}{[n+k]_{q}}\right)\left[\begin{array}{c}
n+k \\
k
\end{array}\right]_{q} x^{k}(1-x)_{q}^{n+1}, \text { if } x \in[0,1), \\
& M_{n, q}(f ; 1)=f(1) \text {, if } x=1, n \in \mathbb{N} .
\end{aligned}
$$

Further, with a slight modification in these operators, Dŏgru and Duman [5] defined the $q$ Meyer-König-Zeller operators for $f \in C[0, a], a \in(0,1)$, as follows:

$$
M_{n, q}(f ; x)=\prod_{s=0}^{n}\left(1-q^{s} x\right) \sum_{k=0}^{\infty} f\left(\frac{q^{n}[k]_{q}}{[n+k]_{q}}\right)\left[\begin{array}{c}
n+k \\
k
\end{array}\right]_{q} x^{k}, q \in(0,1), n \in \mathbb{N} .
$$

Received: June 25, 2018; In revised form: August 6, 2018; Accepted: August 7, 2018

*Corresponding author: R. Maurya; ramapatimaurya@gmail.com

DOI: $10.33205 / \mathrm{cma} .436071$ 
Recently, Gupta and Sharma [9] introduced the Kantorovich type modification of $q$-MeyerKönig-Zeller operators and studied some of their approximation properties. For detail studies of Meyer-König and Zeller operators, one may refer to [6, 7, 10, 16, 17, 20].

In the recent years, $(p, q)$-analogue of various linear positive operators were introduced and studied by many researchers $[1,8,12,15,19]$.

In 2016, Mursaleen et al. [11] introduced the $(p, q)$-Meyer-König-Zeller operators as follows:

$$
M_{n, p, q}(f ; x)=\frac{1}{p^{n(n+1) / 2}} \sum_{k=0}^{\infty}\left[\begin{array}{c}
n+k \\
k
\end{array}\right]_{p, q} x^{k} p^{-k n} \prod_{s=0}^{n}\left(p^{s}-q^{s} x\right) f\left(\frac{p^{n}[k]_{p, q}}{[n+k]_{p, q}}\right) .
$$

Motivated by the above mentioned studied on Meyer-König and Zeller operators, in this paper, we introduced Kantorovich type modification of $(p, q)$-Meyer-König-Zeller operators and discus their approximation properties.

We begin by recalling certain notations of $(p, q)$-calculus (for more details, see [2, 18]).

Let $0<q<p \leq 1$. The $(p, q)$-integer $[n]_{p, q}$ and $(p, q)$-factorial $[n]_{p, q}$ ! are defined by

$$
\begin{gathered}
{[n]_{p, q}=\frac{p^{n}-q^{n}}{p-q}, \quad n=0,1,2 \ldots} \\
{[n]_{p, q} !=\left\{\begin{array}{ll}
{[1]_{p, q}[2]_{p, q} \ldots \ldots \ldots[n]_{p, q},} & \mathrm{n} \geq 1 \\
1, & \mathrm{n}=0
\end{array} .\right.}
\end{gathered}
$$

For integers $0 \leq k \leq n,(p, q)$-binomial coefficient is defined as

$$
\left[\begin{array}{l}
n \\
k
\end{array}\right]_{p, q}=\frac{[n]_{p, q} !}{[k]_{p, q} ![n-k]_{p, q} !} .
$$

The $(p, q)$-binomials expansion is expressed as:

$$
(x+y)_{p, q}^{n}=\prod_{j=0}^{n-1}\left(p^{j} x+q^{j} y\right) .
$$

For a function $f: \mathbb{R} \rightarrow \mathbb{R}$, the $(p, q)$-analogue of derivative is defined as

$$
D_{p, q}(f(x))=\frac{f(p x)-f(q x)}{(p-q) x}, \quad x \neq 0
$$

and

$$
D_{p, q}(f(0))=\lim _{x \rightarrow 0} D_{p, q}(f(x)),
$$

provided the limit exists.

Let $f: C[0, a] \rightarrow R$, the $(p, q)$-integration of a function $f$ is defined as

$$
\int_{0}^{a} f(t) d_{p, q} t=(q-p) a \sum_{k=0}^{\infty} \frac{p^{k}}{q^{k+1}} f\left(\frac{p^{k}}{q^{k+1}} a\right), \text { when } \quad\left|\frac{p}{q}\right|<1,
$$

and

$$
\int_{0}^{a} f(t) d_{p, q} t=(p-q) a \sum_{k=0}^{\infty} \frac{q^{k}}{p^{k+1}} f\left(\frac{q^{k}}{p^{k+1}} a\right), \text { when } \quad\left|\frac{p}{q}\right|>1
$$




\section{CONSTRUCTION OF OPERATORS}

In this section, we introduce the Kantorovich type modification of $(p, q)$-Meyer-König-Zeller operators. We estimate moments and obtain the uniform convergence of operators.

For $0<q<p \leq 1$ and $f \in C[0,1]$, Kantorovich variant of $(p, q)$-Meyer-König-Zeller operators are defined as follows:

$$
\tilde{M}_{n, p, q}(f ; x)=\frac{[n+1]_{p, q}}{p^{n(n-1) / 2}} \sum_{k=0}^{\infty} m_{n, k}^{p, q}(x) \int_{\frac{[k] p_{p, q}}{[n+k]_{p, q}}}^{\frac{[k+1]_{p, q}}{[n+1]_{p, q}}} f\left(p^{n-1} t\right) d_{p, q} t,
$$

where

$$
m_{n, k}^{p, q}(x)=\left[\begin{array}{c}
n+k+1 \\
k
\end{array}\right]_{p, q} p^{-k n} q^{-k} x^{k} P_{n-1}(x),
$$

here, $P_{n}(x)=\prod_{s=0}^{n}\left(p^{s}-q^{s} x\right)$.

Remark 2.1. It can be easily verified that for $p \rightarrow 1$, above operators reduces to $q$-Meyer-König-ZellerKantorovich operators defined in [9].

By using mathematical induction on $n$, one can verify the following identity:

$$
\frac{P_{n}(x)}{p^{n(n+1) / 2}} \sum_{k=0}^{\infty}\left[\begin{array}{c}
n+k \\
k
\end{array}\right]_{p, q} x^{k} p^{-k n}=1 .
$$

Further, by using simple computation, we can obtain the following identity:

$$
\frac{[k+1]_{p, q}}{[n+k+1]_{p, q}}-\frac{[k]_{p, q}}{[n+k]_{p, q}}=\frac{(p q)^{k}[n]_{p, q}}{[n+k]_{p, q}[n+k+1]_{p, q}} .
$$

Lemma 2.1. For $r=0,1,2, \ldots$ and $n>r$, we have

$$
P_{n-1}(x) \sum_{k=0}^{\infty}\left[\begin{array}{c}
n+k-1 \\
k
\end{array}\right]_{p, q} \frac{x^{k} p^{-k(n-r-1)}}{[n+k-1] \frac{r}{p}, q}=\frac{\prod_{j=1}^{r}\left(p^{n-j}-q^{n-j} x\right)}{[n-1]_{\bar{p}, q}^{r}} p^{(n-r)(n-r-1) / 2},
$$

where $[n-1]_{p}^{\frac{r}{p}, q}=[n-1]_{p, q}[n-2]_{p, q} \ldots .[n-r]_{p, q}$.

Proof. By using identity (2.1), lemma can be proved as similar to [9, Lemma 2].

Lemma 2.2. For $r \geq 0$, we have the following inequality

$$
\frac{1}{[n+k+r]_{p, q}} \leq \frac{1}{q^{r+1}[n+k-1]_{p, q}} .
$$

Lemma 2.3. Let $I(f(t))=\int_{\substack{[n+k]_{p}, q \\[n+k, p, q}}^{\frac{[k+1]_{p}, q}{[n]_{p}}} f\left(p^{n-1} t\right) d_{p, q}$ t and $e_{i}=t^{i}$ for $i=0,1,2$, we have following identities:

$$
\begin{aligned}
I\left(e_{0}\right) & =\frac{(p q)^{k}[n]_{p, q}}{[n+k]_{p, q}[n+k+1]_{p, q}}, \\
I\left(e_{1}\right) & =\frac{p^{n-1}}{[2]_{p, q}} \frac{(p q)^{k}[n]_{p, q}}{[n+k]_{p, q}[n+k+1]_{p, q}}\left([k]_{p, q}\left(\frac{q}{[n+k+1]_{p, q}}+\frac{1}{[n+k]_{p, q}}\right)+\frac{p^{k}}{[n+k+1]_{p, q}}\right), \\
I\left(e_{2}\right) & =\frac{p^{2(n-1)}}{[3]_{p, q}} \frac{(p q)^{k}[n]_{p, q}}{[n+k]_{p, q}[n+k+1]_{p, q}}\left([k]_{p, q}^{2} S_{2}(n, k)+[k]_{p, q} S_{1}(n, k)+S_{0}(n, k)\right) .
\end{aligned}
$$


Here,

$$
\begin{aligned}
S_{2}(n, k) & =\frac{q^{2}}{[n+k+1]_{p, q}^{2}}+\frac{q}{[n+k+1]_{p, q}[n+k]_{p, q}}+\frac{1}{[n+k]_{p, q}^{2}}, \\
S_{1}(n, k) & =\frac{2 p^{k} q}{[n+k+1]_{p, q}^{2}}+\frac{p^{k}}{[n+k+1]_{p, q}[n+k]_{p, q}}, \\
S_{0}(n, k) & =\frac{p^{2 k}}{[n+k+1]_{p, q}^{2}} .
\end{aligned}
$$

Proof. By using definition of $(p, q)$-integral and identity (2.2), we have

$$
\begin{aligned}
& I\left(e_{2}\right)=\int_{\frac{[k]_{p, q}}{[n+k]_{p, q}}}^{\frac{[k+1]_{p, q}}{[n+k+1]_{p, q}}}\left(p^{n-1} t\right)^{2} d_{p, q} t \\
& =p^{2(n-1)}(p-q)\left(\left(\frac{[k+1]_{p, q}}{[n+k+1]_{p, q}}\right)^{3}-\left(\frac{[k]_{p, q}}{[n+k]_{p, q}}\right)^{3}\right) \sum_{j=0}^{\infty}\left(\frac{q^{j}}{p^{j+1}}\right)^{3} \\
& =\frac{p^{2(n-1)}}{[3]_{p, q}} \frac{(p q)^{k}[n]_{p, q}}{[n+k]_{p, q}[n+k+1]_{p, q}} \\
& \left(\frac{[k+1]_{p, q}^{2}}{[n+k+1]_{p, q}^{2}}+\frac{[k+1]_{p, q}[k]_{p, q}}{[n+k+1]_{p, q}[n+k]_{p, q}}+\frac{[k]_{p, q}^{2}}{[n+k]_{p, q}^{2}}\right) \\
& =\frac{p^{2(n-1)}}{[3]_{p, q}} \frac{(p q)^{k}[n]_{p, q}}{[n+k]_{p, q}[n+k+1]_{p, q}} \\
& \left(\frac{\left(p^{k}+q[k]_{p, q}\right)^{2}}{[n+k+1]_{p, q}^{2}}+\frac{\left(p^{k}+q[k]_{p, q}\right)[k]_{p, q}}{[n+k+1]_{p, q}[n+k]_{p, q}}+\frac{[k]_{p, q}^{2}}{[n+k]_{p, q}^{2}}\right) \\
& =\frac{p^{2(n-1)}}{[3]_{p, q}} \frac{(p q)^{k}[n]_{p, q}}{[n+k]_{p, q}[n+k+1]_{p, q}} \\
& \left(\frac{\left(p^{2 k}+2 p^{k} q[k]_{p, q}+q^{2}[k]_{p, q}^{2}\right)}{[n+k+1]_{p, q}^{2}}+\frac{\left(p^{k}[k]_{p, q}+q[k]_{p, q}^{2}\right)}{[n+k+1]_{p, q}[n+k]_{p, q}}+\frac{[k]_{p, q}^{2}}{[n+k]_{p, q}^{2}}\right) \\
& =\frac{p^{2(n-1)}}{[3]_{p, q}} \frac{(p q)^{k}[n]_{p, q}}{[n+k]_{p, q}[n+k+1]_{p, q}}\left([k]_{p, q}^{2} S_{2}(n, k)+[k]_{p, q} S_{1}(n, k)+S_{0}(n, k)\right) \text {. }
\end{aligned}
$$

Similarly, we can get result for $e_{0}$ and $e_{1}$.

Lemma 2.4. For $e_{i}=t^{i}$, here $i=0,1,2$, moments estimate of proposed operators are as follows:

$$
\begin{aligned}
& \tilde{M}_{n, p, q}\left(e_{0} ; x\right)=1, \\
& \tilde{M}_{n, p, q}\left(e_{1} ; x\right) \leq \frac{1}{[2]_{p, q} q}\left(2 x+\frac{\left(p^{n-1}-q^{n-1} x\right)}{q[n-1]_{p, q}}\right), \\
& \tilde{M}_{n, p, q}\left(e_{1} ; x\right) \geq \frac{2 x}{[2]_{p, q} q^{2}}\left(1-\frac{(1+q)}{p^{n-1}}\left(\frac{p^{n-1}-q^{n-1} x}{[n-1]_{p, q}}\right)\right), \\
& \tilde{M}_{n, p, q}\left(e_{2} ; x\right) \leq \frac{1}{[3]_{p, q} q}\left(3 x^{2}+\left(\frac{3 x}{q}+\frac{3}{q^{2}}+\frac{p}{q^{3}} \frac{\left(p^{n-2}-q^{n-2} x\right)}{[n-2]_{p, q}}\right) \frac{\left(p^{n-1}-q^{n-1} x\right)}{[n-1]_{p, q}}\right) .
\end{aligned}
$$


Proof. By using definition of $(p, q)$-Meyer-König-Zeller Kantorovich operators, identity (2.1), Lemma 2.2 and Lemma 2.3, moments of sequence of the opeartors can be estimated as follows:

For $e_{0}=1$, we have

$$
\begin{aligned}
\tilde{M}_{n, p, q}\left(e_{0} ; x\right)= & \frac{[n+1]_{p, q}}{p^{n(n-1) / 2}} P_{n-1}(x) \sum_{k=0}^{\infty} m_{n, k}^{p, q}(x) \frac{(p q)^{k}[n]_{p, q}}{[n+k]_{p, q}[n+k+1]_{p, q}} \\
= & \frac{[n+1]_{p, q}}{p^{n(n-1) / 2} P_{n-1}(x) \sum_{k=0}^{\infty}\left[\begin{array}{c}
n+k+1 \\
k
\end{array}\right]_{p, q} x^{k} p^{-k n} q^{-k}} \\
& \frac{(p q)^{k}[n]_{p, q}}{[n+k]_{p, q}[n+k+1]_{p, q}} \\
= & \frac{P_{n-1}(x)}{p^{n(n-1) / 2}} \sum_{k=0}^{\infty}\left[\begin{array}{c}
n+k-1 \\
=
\end{array}\right]_{p, q} x^{k} p^{-k(n-1)} \\
= & 1 .
\end{aligned}
$$

For $e_{1}=t$, upper bound of moment can be obtained as follows:

$$
\begin{aligned}
& \tilde{M}_{n, p, q}\left(e_{1} ; x\right) \leq \frac{[n+1]_{p, q}}{p^{n(n-1) / 2}} P_{n-1}(x) \sum_{k=0}^{\infty}\left[\begin{array}{c}
n+k+1 \\
k
\end{array}\right]_{p, q} x^{k} p^{-k n} q^{-k} \\
& \frac{p^{n-1}}{[2]_{p, q}} \frac{(p q)^{k}[n]_{p, q}}{[n+k]_{p, q}[n+k+1]_{p, q}}\left(\frac{[k]_{p, q}}{q} \frac{2}{[n+k-1]_{p, q}}+\frac{p^{k}}{q^{2}[n+k-1]_{p, q}}\right) \\
& =\frac{p^{n-1}}{[2]_{p, q} q p^{n(n-1) / 2}}\left(2 P_{n-1}(x) \sum_{k=0}^{\infty}\left[\begin{array}{c}
n+k-1 \\
k
\end{array}\right]_{p, q} x^{k} p^{-k(n-1)} \frac{[k]_{p, q}}{[n+k-1]_{p, q}}\right. \\
& \left.+\frac{P_{n-1}(x)}{q} \sum_{k=0}^{\infty}\left[\begin{array}{c}
n+k-1 \\
k
\end{array}\right]_{p, q} \frac{x^{k} p^{-k(n-2)}}{[n+k-1]_{p, q}}\right) \\
& =\frac{p^{n-1}}{[2]_{p, q} q p^{n(n-1) / 2}}\left(2 P_{n-1}(x) \sum_{k=1}^{\infty}\left[\begin{array}{c}
n+k-2 \\
k-1
\end{array}\right]_{p, q} x^{k} p^{-k(n-1)}\right. \\
& \left.+\frac{P_{n-1}(x)}{q} \sum_{k=0}^{\infty}\left[\begin{array}{c}
n+k-1 \\
k
\end{array}\right]_{p, q} \frac{x^{k} p^{-k(n-2)}}{[n+k-1]_{p, q}}\right) \\
& =\frac{p^{n-1}}{[2]_{p, q} q p^{n(n-1) / 2}}\left(\frac{2 x}{p^{(n-1)}} P_{n-1}(x) \sum_{k=0}^{\infty}\left[\begin{array}{c}
n+k-1 \\
k
\end{array}\right]_{p, q} x^{k} p^{-k(n-1)}\right. \\
& \left.+\frac{P_{n-1}(x)}{q} \sum_{k=0}^{\infty}\left[\begin{array}{c}
n+k-1 \\
k
\end{array}\right]_{p, q} \frac{x^{k} p^{-k(n-2)}}{[n+k-1]_{p, q}}\right) \\
& =\frac{1}{[2]_{p, q} q}\left(2 x+\frac{p^{n-1}-q^{n-1} x}{q[n-1]_{p, q}}\right) \text {. }
\end{aligned}
$$


Lower bound of moment for $e_{1}$ can be obtained as follows:

$$
\begin{aligned}
& \tilde{M}_{n, p, q}\left(e_{1} ; x\right)=\frac{p^{n-1} P_{n-1}(x)}{[2]_{p, q} p^{n(n-1) / 2}} \sum_{k=0}^{\infty}\left[\begin{array}{c}
n+k-1 \\
k
\end{array}\right]_{p, q} x^{k} p^{-k(n-1)}\left(\frac{[k+1]_{p, q}}{[n+k+1]_{p, q}}+\frac{[k]_{p, q}}{[n+k]_{p, q}}\right) \\
& =\frac{p^{n-1} P_{n-1}(x)}{[2]_{p, q} p^{n(n-1) / 2}} \sum_{k=1}^{\infty}\left[\begin{array}{c}
n+k-2 \\
k-1
\end{array}\right]_{p, q} x^{k} p^{-k(n-1)} \\
& \frac{[n+k-1]_{p, q}}{[k]_{p, q}}\left(\frac{[k+1]_{p, q}}{[n+k+1]_{p, q}}+\frac{[k]_{p, q}}{[n+k]_{p, q}}\right) \\
& =\frac{p^{n-1} P_{n-1}(x)}{[2]_{p, q} p^{n(n-1) / 2}} \sum_{k=0}^{\infty}\left[\begin{array}{c}
n+k-1 \\
k
\end{array}\right]_{p, q} x^{k+1} p^{-(k+1)(n-1)} \\
& \frac{[n+k]_{p, q}}{[k+1]_{p, q}}\left(\frac{[k+2]_{p, q}}{[n+k+2]_{p, q}}+\frac{[k+1]_{p, q}}{[n+k+1]_{p, q}}\right) \\
& \geq \frac{x P_{n-1}(x)}{[2]_{p, q} p^{n(n-1) / 2}} \sum_{k=0}^{\infty}\left[\begin{array}{c}
n+k-1 \\
k
\end{array}\right]_{p, q} x^{k} p^{-k(n-1)} \\
& {[n+k]_{p, q}\left(\frac{1}{[n+k+2]_{p, q}}+\frac{1}{[n+k+1]_{p, q}}\right)} \\
& \geq \frac{2 x P_{n-1}(x)}{[2]_{p, q} p^{n(n-1) / 2}} \sum_{k=0}^{\infty}\left[\begin{array}{c}
n+k-1 \\
k
\end{array}\right]_{p, q} x^{k} p^{-k(n-1)}\left(\frac{[n+k]_{p, q}}{[n+k+2]_{p, q}}\right) \\
& =\frac{2 x P_{n-1}(x)}{[2]_{p, q} p^{n(n-1) / 2}} \sum_{k=0}^{\infty}\left[\begin{array}{c}
n+k-1 \\
k
\end{array}\right]_{p, q} x^{k} p^{-k(n-1)} \\
& \left(\frac{[n+k+2]_{p, q}-q p^{n+k}-p^{n+k+1}}{q^{2}[n+k+2]_{p, q}}\right) \\
& \geq \frac{2 x P_{n-1}(x)}{[2]_{p, q} p^{n(n-1) / 2}} \sum_{k=0}^{\infty}\left[\begin{array}{c}
n+k-1 \\
k
\end{array}\right]_{p, q} x^{k} p^{-k(n-1)} \\
& \left(\frac{1}{q^{2}}-\frac{p^{k}}{q[n+k-1]_{p, q}}-\frac{p^{k}}{q^{2}[n+k-1]_{p, q}}\right) \\
& =\frac{2 x P_{n-1}(x)}{[2]_{p, q} p^{n(n-1) / 2}} \sum_{k=0}^{\infty}\left[\begin{array}{c}
n+k-1 \\
k
\end{array}\right]_{p, q} x^{k} p^{-k(n-1)} \\
& \left(\frac{1}{q^{2}}-\left(\frac{1}{q}+\frac{1}{q^{2}}\right) \frac{p^{k}}{[n+k-1]_{p, q}}\right) \\
& =\frac{2 x}{[2]_{p, q} q^{2} p^{n(n-1) / 2}} P_{n-1}(x) \sum_{k=0}^{\infty}\left[\begin{array}{c}
n+k-1 \\
k
\end{array}\right]_{p, q} x^{k} p^{-k(n-1)} \\
& -\frac{2(1+q) x}{[2]_{p, q} q^{2} p^{n(n-1) / 2}} P_{n-1}(x) \sum_{k=0}^{\infty}\left[\begin{array}{c}
n+k-1 \\
k
\end{array}\right]_{p, q} x^{k} p^{-k(n-2)} \frac{1}{[n+k-1]_{p, q}}
\end{aligned}
$$




$$
\begin{aligned}
& =\frac{2 x}{[2]_{p, q} q^{2}}-\frac{2(1+q) x}{[2]_{p, q} q^{2} p^{n-1}}\left(\frac{p^{n-1}-q^{n-1} x}{[n-1]_{p, q}}\right) \\
& =\frac{2 x}{[2]_{p, q} q^{2}}\left(1-\frac{(1+q)}{p^{n-1}}\left(\frac{p^{n-1}-q^{n-1} x}{[n-1]_{p, q}}\right)\right) .
\end{aligned}
$$

Finally for $e_{2}=t^{2}$, moments of the operators can be obtained as follows:

$$
\tilde{M}_{n, p, q}\left(e_{2} ; x\right)=A+B+C \text {. }
$$

Here,

$$
\begin{aligned}
A & =\frac{p^{2(n-1)}}{[3]_{p, q} p^{n(n-1) / 2}} P_{n-1}(x) \sum_{k=0}^{\infty}\left[\begin{array}{c}
n+k-1 \\
k
\end{array}\right]_{p, q} x^{k} p^{-k(n-1)}[k]_{p, q}^{2} S_{2}(n, k), \\
B & =\frac{p^{2(n-1)}}{[3]_{p, q} p^{n(n-1) / 2}} P_{n-1}(x) \sum_{k=0}^{\infty}\left[\begin{array}{c}
n+k-1 \\
k
\end{array}\right]_{p, q} x^{k} p^{-k(n-1)}[k]_{p, q} S_{1}(n, k), \\
C & =\frac{p^{2(n-1)}}{[3]_{p, q} p^{n(n-1) / 2}} P_{n-1}(x) \sum_{k=0}^{\infty}\left[\begin{array}{c}
n+k-1 \\
k
\end{array}\right]_{p, q} x^{k} p^{-k(n-1)} S_{0}(n, k) .
\end{aligned}
$$

Using Lemma 2.2, we have

$$
\begin{aligned}
& S_{2}(n, k) \leq \frac{3}{q^{3}[n+k-1] \frac{2}{p}, q} \\
& S_{1}(n, k) \leq \frac{3 p^{k}}{q^{4}[n+k-1] \frac{2}{p}, q} \\
& S_{0}(n, k) \leq \frac{p^{2 k}}{q^{5}[n+k-1] \frac{2}{p}, q} .
\end{aligned}
$$

Using the inequality (2.3), we have

$$
\begin{aligned}
A & \leq \frac{3 p^{2(n-1)}}{[3]_{p, q} q^{3} p^{n(n-1) / 2}} P_{n-1}(x) \sum_{k=0}^{\infty}\left[\begin{array}{c}
n+k-1 \\
k
\end{array}\right]_{p, q} x^{k} p^{-k(n-1)} \frac{[k]_{p, q}^{2}}{[n+k-1]_{p, q}^{2}} \\
& =\frac{3 p^{2(n-1)}}{[3]_{p, q} q^{3} p^{n(n-1) / 2}} P_{n-1}(x) \sum_{k=1}^{\infty}\left[\begin{array}{c}
n+k-2 \\
k-1
\end{array}\right]_{p, q} x^{k} p^{-k(n-1)} \frac{[k]_{p, q}}{[n+k-2]_{p, q}} \\
& =\frac{3 p^{2(n-1)}}{[3]_{p, q} q^{3} p^{n(n-1) / 2}} P_{n-1}(x) \sum_{k=0}^{\infty}\left[\begin{array}{c}
n+k-1 \\
k
\end{array}\right]_{p, q} x^{k+1} p^{-(k+1)(n-1)} \frac{[k+1]_{p, q}}{[n+k-1]_{p, q}} \\
& =\frac{3 p^{2(n-1)}}{[3]_{p, q} q^{3} p^{n(n-1) / 2}} P_{n-1}(x)\left(\sum_{k=0}^{\infty}\left[\begin{array}{c}
n+k-1 \\
k
\end{array}\right]_{p, q} x^{k+1} p^{-(k+1)(n-1)} \frac{p^{k}}{[n+k-1]_{p, q}}\right. \\
& \left.\sum_{k=0}^{\infty}\left[\begin{array}{c}
n+k-1 \\
k
\end{array}\right]_{p, q} x^{k+1} p^{-(k+1)(n-1)} \frac{q[k]_{p, q}}{[n+k-1]_{p, q}}\right)
\end{aligned}
$$




$$
\begin{aligned}
& =\frac{3 p^{2(n-1)}}{[3]_{p, q} q^{3} p^{n(n-1) / 2}}\left(x p^{-(n-1)} P_{n-1}(x) \sum_{k=0}^{\infty}\left[\begin{array}{c}
n+k-1 \\
k
\end{array}\right]_{p, q} \frac{x^{k} p^{-k(n-2)}}{[n+k-1]_{p, q}}\right. \\
& \left.+q p^{-2(n-1)} x^{2} P_{n-1}(x) \sum_{k=0}^{\infty}\left[\begin{array}{c}
n+k-1 \\
k
\end{array}\right]_{p, q} x^{k} p^{-k(n-1)}\right) \\
& =\frac{3 p^{2(n-1)}}{[3]_{p, q} q^{3} p^{n(n-1) / 2}}\left(x p^{-(n-1)} p^{(n-1)(n-2) / 2} \frac{\left(p^{n-1}-q^{n-1} x\right)}{[n-1]_{p, q}}+q p^{-2(n-1)} p^{n(n-1) / 2} x^{2}\right) \\
& =\frac{3 x}{[3]_{p, q} q^{3}} \frac{\left(p^{n-1}-q^{n-1} x\right)}{[n-1]_{p, q}}+\frac{3 x^{2}}{[3]_{p, q} q^{2}} .
\end{aligned}
$$

Again, using the inequality (2.4), we have

$$
\begin{aligned}
B & \leq \frac{3 p^{2(n-1)}}{[3]_{p, q} q^{4} p^{n(n-1) / 2}} P_{n-1}(x) \sum_{k=1}^{\infty}\left[\begin{array}{c}
n+k-2 \\
k-1
\end{array}\right]_{p, q} x^{k} p^{-k(n-1)} \frac{p^{k}}{[n+k-2]_{p, q}} \\
& =\frac{3 p^{2(n-1)}}{[3]_{p, q} q^{4} p^{n(n-1) / 2}} P_{n-1}(x) \sum_{k=0}^{\infty}\left[\begin{array}{c}
n+k-1 \\
k
\end{array}\right]_{p, q} x^{k+1} p^{-(k+1)(n-1)} \frac{p^{k+1}}{[n+k-1]_{p, q}} \\
& =\frac{3 p^{2(n-1)}}{[3]_{p, q} q^{4} p^{n(n-1) / 2}}\left(x p^{-(n-1)} p^{(n-1)(n-2) / 2} \frac{\left(p^{n-1}-q^{n-1} x\right)}{[n-1]_{p, q}}\right) \\
& =\frac{3}{[3]_{p, q} q^{4}} \frac{\left(p^{n-1}-q^{n-1} x\right)}{[n-1]_{p, q}} .
\end{aligned}
$$

Further, using the inequality (2.5), we have

$$
\begin{aligned}
C & \leq \frac{p^{2(n-1)}}{[3]_{p, q} q^{5} p^{n(n-1) / 2}} P_{n-1}(x) \sum_{k=0}^{\infty}\left[\begin{array}{c}
n+k-1 \\
k
\end{array}\right]_{p, q} \frac{x^{k} p^{-k(n-3)}}{[n+k-1]^{\frac{2}{p}, q}} \\
& =\frac{p}{[3]_{p, q} q^{5}} \frac{\prod_{j=1}^{2}\left(p^{n-j}-q^{n-j} x\right)}{[n-1]_{\frac{2}{p}, q}^{2}} .
\end{aligned}
$$

Finally,

$$
\tilde{M}_{n, p, q}\left(e_{2} ; x\right) \leq \frac{1}{[3]_{p, q} q^{2}}\left(3 x^{2}+\left(\frac{3 x}{q}+\frac{3}{q^{2}}+\frac{p}{q^{3}} \frac{\left(p^{n-2}-q^{n-2} x\right)}{[n-2]_{p, q}}\right) \frac{\left(p^{n-1}-q^{n-1} x\right)}{[n-1]_{p, q}}\right) .
$$

Hence the lemma.

Lemma 2.5. For all $x \in[0,1]$ and $0<q<p \leq 1$, central moments of the operators are given by:

$$
\begin{aligned}
\tilde{M}_{n, p, q}((t-x) ; x) \leq & \frac{1}{[2]_{p, q} q}\left(\left(2-[2]_{p, q} q\right) x+\frac{\left(p^{n-1}-q^{n-1} x\right)}{q[n-1]_{p, q}}\right), \\
\tilde{M}_{n, p, q}\left((t-x)^{2} ; x\right) \leq & \left(1+\frac{3}{[3]_{p, q} q^{2}}-\frac{4}{[2]_{p, q} q^{2}}\right) x^{2}+\frac{p^{n-1}-q^{n-1} x}{[n-1]_{p, q} q^{2}} \\
& \left(\frac{3 x}{[3]_{p, q}}+\frac{3}{[3]_{p, q} q^{2}}+\frac{4 x^{2}(1+q)}{[2]_{p, q} p^{n-1}}+\frac{p}{q^{3}} \frac{p^{n-2}-q^{n-2} x}{[n-2]_{p, q}}\right) .
\end{aligned}
$$

Now, we give the result for the uniform convergence of operator by means of BohmanKorovkin type theorem. 
Remark 2.2. For $0<q<p \leq 1$, by simple computations $\lim _{n \rightarrow \infty}[n]_{p, q}=1 /(p-q)$. In order to obtain results for order of convergence of the operator, we take $q_{n} \in(0,1), p_{n} \in\left(q_{n}, 1\right]$ such that $\lim _{n \rightarrow \infty} p_{n}=\lim _{n \rightarrow \infty} q_{n}=1, \lim _{n \rightarrow \infty} p_{n}^{n}=a$ and $\lim _{n \rightarrow \infty} q_{n}^{n}=b$, so that $\lim _{n \rightarrow \infty} \frac{1}{[n]_{p_{n}, q_{n}}}=0$. Such a sequence can always be constructed for example, we can take $q_{n}=1-1 / n$ and $p_{n}=1-1 / 2 n$, clearly $\lim _{n \rightarrow \infty} p_{n}^{n}=e^{-1 / 2}, \lim _{n \rightarrow \infty} q_{n}^{n}=e^{-1}$ and $\lim _{n \rightarrow \infty} \frac{1}{[n]_{p_{n}, q_{n}}}=0$.

Theorem 2.1. Let $\left\{p_{n}\right\}_{n}$ and $\left\{q_{n}\right\}_{n}$ be the sequence as defined in Remark 2.2. Then for each $f \in$ $C[0,1], \tilde{M}_{n, p_{n}, q_{n}}(f ; x)$ converges uniformly to $f$ on $[0,1]$.

Proof. By the Bohman-Korovkin theorem [13], to prove uniform convergence of the operators, it is sufficient to show that following equality holds for $i=0,1,2$ :

$$
\lim _{n \rightarrow \infty}\left\|\tilde{M}_{n, p_{n}, q_{n}}\left(e_{i} ; .\right)-e_{i}\right\|=0 .
$$

By using moment estimates obtained in Lemma 2.4, equality (2.6) holds directly for $i=0$. Also,

$$
\begin{aligned}
\left\|\tilde{M}_{n, p_{n}, q_{n}}\left(e_{1} ; .\right)-e_{1}\right\| \leq & \frac{1}{[2]_{p_{n}, q_{n}} q_{n}}\left(\left|2-[2]_{p_{n}, q_{n}} q_{n}\right|+\left|\frac{\left(p_{n}^{n-1}-q_{n}^{n-1} x\right)}{q_{n}[n-1]_{p_{n}, q_{n}}}\right|\right), \\
\left\|\tilde{M}_{n, p_{n}, q_{n}}\left(e_{2} ; .\right)-e_{2}\right\| \leq & \frac{1}{[3]_{p_{n}, q_{n}} q_{n}^{2}}\left(\left|3-[3]_{p_{n}, q_{n}} q_{n}^{2}\right|\right. \\
& \left.+\left|\left(\frac{3}{q_{n}}+\frac{3}{q_{n}^{2}}+\frac{p_{n}}{q_{n}^{3}} \frac{\left(p_{n}^{n-2}-q_{n}^{n-2} x\right)}{[n-2]_{p_{n}, q_{n}}}\right) \frac{\left(p_{n}^{n-1}-q_{n}^{n-1} x\right)}{[n-1]_{p_{n}, q_{n}}}\right|\right) .
\end{aligned}
$$

For $n \rightarrow \infty$ and Remark 2.2, equality (2.6) holds for $i=0,1,2$. Hence the theorem.

\section{RATE OF CONVERGENCE}

In this section, we estimate the rate of convergence of proposed operator by means of modulus of continuity and Lipschitz class functions. We also show the statistical convergence of the operator.

Recall the concept of modulus of continuity, the modulus of continuity of $f(x) \in[0, a]$, denoted by $\omega(f, \delta)$, is defined by

$$
\omega(f, \delta)=\sup _{|x-y| \leq \delta ; x, y \in[0, a]}|f(x)-f(y)| .
$$

A function $f \in \operatorname{Lip}_{M}(\alpha),(M>0$ and $0<\alpha \leq 1)$, if the inequality

$$
|f(t)-f(x)| \leq M|t-x|^{\alpha},
$$

holds for all $t, x \in[0,1]$.

Theorem 3.2. Let $\left\{p_{n}\right\}_{n}$ and $\left\{q_{n}\right\}_{n}$ be the sequence as defined in Remark 2.2. Then

$$
\left|\tilde{M}_{n, p_{n}, q_{n}}(f ; x)-f\right| \leq 2 \omega\left(f, \sqrt{ } \delta_{n}\right),
$$

for all $f \in C[0,1]$, here $\delta_{n}=\tilde{M}_{n, p_{n}, q_{n}}\left((t-x)^{2} ; x\right)$.

Proof. By the linearity and monotonicity of the operators, we get

$$
\left|\tilde{M}_{n, p_{n}, q_{n}}(f ; x)-f\right| \leq \tilde{M}_{n, p_{n}, q_{n}}(|f(t)-f(x)| ; x),
$$

also, by property of modulus of continuity (see [14])

$$
|f(t)-f(x)| \leq \omega(f, \delta)\left(1+\frac{1}{\delta^{2}}(t-x)^{2}\right),
$$


therefore,

$$
\left|\tilde{M}_{n, p_{n}, q_{n}}(f ; x)-f\right| \leq \omega(f, \delta)\left(1+\frac{1}{\delta^{2}} \tilde{M}_{n, p_{n}, q_{n}}\left((t-x)^{2} ; x\right)\right) .
$$

By Lemma 2.5 and Remark 2.2, we can find

$$
\lim _{n \rightarrow \infty} \tilde{M}_{n, p_{n}, q_{n}}\left((t-x)^{2} ; x\right)=0 .
$$

So, letting $\delta_{n}=\tilde{M}_{n, p_{n}, q_{n}}\left((t-x)^{2} ; x\right)$ and take $\delta=\sqrt{ } \delta_{n}$, we finally get the result.

In the following theorem, we compute the rate of convergence by means of the Lipschitz class.

Theorem 3.3. Let $\left\{p_{n}\right\}_{n}$ and $\left\{q_{n}\right\}_{n}$ be the sequence as defined in Remark 2.2. Then for all $f \in$ $\operatorname{Lip}_{M}(\alpha)$, we have

$$
\left|\tilde{M}_{n, p_{n}, q_{n}}(f ; x)-f(x)\right| \leq M \delta_{n}(x)^{\alpha / 2},
$$

here $\delta_{n}(x)=\tilde{M}_{n, p_{n}, q_{n}}\left(|t-x|^{2} ; x\right)$.

Proof. By using definition of Lipschitz class functions and applying Hölder's inequality with $p=\frac{2}{\alpha}, q=\frac{2}{2-\alpha}$, we get

$$
\begin{aligned}
\left|\tilde{M}_{n, p_{n}, q_{n}}(f ; x)-f(x)\right| & \leq \tilde{M}_{n, p_{n}, q_{n}}(|f(t)-f(x)| ; x) \\
& \leq M \tilde{M}_{n, p_{n}, q_{n}}\left(|t-x|^{\alpha} ; x\right) \\
& \leq M \tilde{M}_{n, p_{n}, q_{n}}\left(|t-x|^{2} ; x\right)^{\alpha / 2}
\end{aligned}
$$

Taking $\delta_{n}=\tilde{M}_{n, p_{n}, q_{n}}\left(|t-x|^{2} ; x\right)$, we get the result.

A sequence $\left(x_{n}\right)_{n}$ is said to be statistically convergent to a number $L$, denoted by $s t-$ $\lim _{n} x_{n}=L$ if, for every $\varepsilon>0$,

$$
\delta\left\{n \in \mathbb{N}:\left|x_{n}-L\right| \geq \varepsilon\right\}=0,
$$

where

$$
\delta(S):=\frac{1}{N} \sum_{k=1}^{N} \chi_{S}(j)
$$

is the natural density of set $S \subseteq \mathbb{N}$ and $\chi_{S}$ is the characteristic function of $S$.

Let $C_{B}(D)$ represents the space of all continuous functions on $\mathrm{D}$ and bounded on entire real line, where $\mathrm{D}$ is any interval on real line. It can be easily shown that $C_{B}(D)$ is a Banach space with supreme norm.

Theorem A. ([5]) Let $\left\{L_{n}\right\}_{n}$ be a sequence of positive linear operators from $C_{B}([a, b])$ into $B([a, b])$, satisfying the condition that

$$
s t-\lim _{n \rightarrow \infty}\left\|L_{n} e_{i}-e_{i}\right\|=0 \forall i=0,1,2 .
$$

Then,

$$
s t-\lim _{n \rightarrow \infty}\left\|L_{n} f-f\right\|=0 \forall f \in C_{B}([a, b]) .
$$


Theorem 3.4. Let $\left\{p_{n}\right\}_{n},\left\{q_{n}\right\}_{n}$ be sequences such that

. Then, we have

$$
\begin{aligned}
& s t-\lim _{n \rightarrow \infty} q_{n}=1, s t-\lim _{n \rightarrow \infty} q_{n}{ }^{n}=a, \\
& s t-\lim _{n \rightarrow \infty} p_{n}=1, s t-\lim _{n \rightarrow \infty} p_{n}{ }^{n}=b .
\end{aligned}
$$

$$
s t-\lim _{n \rightarrow \infty}\left\|\tilde{M}_{n, p_{n}, q_{n}} f-f\right\|=0 \text { for all } f \in C_{B}[0,1] \text {. }
$$

Proof. We use moment estimates obtained in Lemma 2.4, to prove that operator converges statistically for $e_{i}, i=0,1,2$. For first moment result is trivial.

For $i=1,2$, we have

$$
\begin{aligned}
& \left|\tilde{M}_{n, p_{n}, q_{n}}\left(e_{1} ; .\right)-e_{1}\right| \leq \frac{1}{[2]_{p_{n}, q_{n}} q_{n}}\left(\left|2-[2]_{p_{n}, q_{n}} q_{n}\right| x+\left|\frac{\left(p_{n}^{n-1}-q_{n}^{n-1} x\right)}{q_{n}[n-1]_{p_{n}, q_{n}}}\right|\right), \\
& \left|\tilde{M}_{n, p_{n}, q_{n}}\left(e_{2} ; .\right)-e_{2}\right| \leq \frac{1}{[3]_{p_{n}, q_{n}} q_{n}^{2}}\left(\left|3-[3]_{p_{n}, q_{n}} q_{n}^{2}\right| x^{2}+\left|\left(\frac{3 x}{q_{n}}+\frac{3}{q_{n}^{2}}\right) \frac{\left(p_{n}^{n-1}-q_{n}^{n-1} x\right)}{[n-1]_{p_{n}, q_{n}}}\right|\right. \\
& \left.+\left|\frac{p_{n}}{q_{n}^{3}} \frac{\left(p_{n}^{n-1}-q_{n}^{n-1} x\right)\left(p_{n}^{n-2}-q_{n}^{n-2} x\right)}{[n-1]_{p_{n}, q_{n}}[n-2]_{p_{n}, q_{n}}}\right|\right) .
\end{aligned}
$$

By taking supremum over $x \in[0,1]$ in above inequalities and using $s t-\lim _{n \rightarrow \infty} \frac{1}{[n]_{p_{n}, q_{n}}}=0$, we get

$$
\begin{aligned}
& s t-\lim _{n \rightarrow \infty}\left\|\tilde{M}_{n, p_{n}, q_{n}}\left(e_{1} ; .\right)-e_{1}\right\|=0, \\
& s t-\lim _{n \rightarrow \infty}\left\|\tilde{M}_{n, p_{n}, q_{n}}\left(e_{2} ; .\right)-e_{2}\right\|=0 .
\end{aligned}
$$

By Theorem A, we obtain statistical convergence of the operator.

\section{LOCAL APPROXIMATION}

The Peetre's $K$-functional is defined by

$$
K_{2}(f, \delta)=\inf _{g \in W^{2}}\left\{\|f-g\|+\delta\left\|g^{\prime \prime}\right\|\right\},
$$

here $W^{2}=\left\{g \in C[0,1]: g^{\prime}, g^{\prime \prime} \in C[0,1]\right\}$ and norm $\|$.$\| denotes the uniform norm on C[0,1]$. Further, we have a well-known inequality given by DeVore and Lorentz [4, p. 177, Theorem $2.4]$, there exists a positive constant $C>0$ such that $K_{2}(f, \delta) \leq C \omega\left(f, \delta^{\frac{1}{2}}\right), \delta>0$, where $\omega_{2}$ is known as the second order modulus of continuity, given by

$$
\omega_{2}\left(f, \delta^{\frac{1}{2}}\right)=\sup _{0<h<\delta^{\frac{1}{2}}, x \in[0,1]}|f(x+2 h)-2 f(x+h)+f(x)| .
$$

Here, we give some local result for the operators.

Theorem 4.5. Let $\left\{p_{n}\right\}_{n}$ and $\left\{q_{n}\right\}_{n}$ be the sequence as defined in Remark 2.2. Then for all $f \in C[0,1]$, there exists an absolute constant $C>0$ such that

$$
\left|\tilde{M}_{n, p_{n}, q_{n}}(f ; x)-f\right| \leq C \omega_{2}\left(f, \delta_{n}(x)\right)+\omega\left(f, \alpha_{n}(x)\right),
$$

Here,

$$
\begin{gathered}
\delta_{n}(x)=\sqrt{\tilde{M}_{n, p_{n}, q_{n}}\left((t-x)^{2} ; x\right)+\left(\frac{1}{[2]_{p_{n}, q_{n}} q_{n}}\left(2 x+\frac{\left(p_{n}^{n-1}-q_{n}^{n-1} x\right)}{q_{n}[n-1]_{p_{n}, q_{n}}}\right)-x\right)^{2}}, \\
\alpha_{n}(x)=\left|\frac{1}{[2]_{p_{n}, q_{n}} q_{n}}\left(\left(2-[2]_{p_{n}, q_{n}} q_{n}\right) x+\frac{\left(p_{n}^{n-1}-q_{n}^{n-1} x\right)}{q_{n}[n-1]_{p_{n}, q_{n}}}\right)\right| .
\end{gathered}
$$


Proof. For $f \in C[0,1]$, we consider

$$
M_{n, p_{n}, q_{n}}(f ; x)=\tilde{M}_{n, p_{n}, q_{n}}(f ; x)+f(x)-f\left(\frac{1}{[2]_{p_{n}, q_{n}} q_{n}}\left(2 x+\frac{\left(p_{n}^{n-1}-q_{n}^{n-1} x\right)}{q_{n}[n-1]_{p_{n}, q_{n}}}\right)\right) .
$$

Now, using Lemma 2.4, we immediately get

$$
M_{n, p_{n}, q_{n}}(1 ; x)=\tilde{M}_{n, p_{n}, q_{n}}(1 ; x)=1
$$

and

$$
M_{n, p_{n}, q_{n}}(t ; x)=\tilde{M}_{n, p_{n}, q_{n}}(t ; x)+x-\frac{1}{[2]_{p_{n}, q_{n}} q_{n}}\left(2 x+\frac{\left(p_{n}^{n-1}-q_{n}^{n-1} x\right)}{q_{n}[n-1]_{p_{n}, q_{n}}}\right) \leq x .
$$

By Taylor's formula $g(t)=g(x)+(t-x) g^{\prime}(x)+\int_{x}^{t}(t-u) g^{\prime \prime}(u) d u$, we get

$$
\begin{aligned}
M_{n, p_{n}, q_{n}}(g(t) ; x)= & g(x)+g^{\prime}(x) M_{n, p_{n}, q_{n}}((t-x) ; x)+M_{n, p_{n}, q_{n}}\left(\int_{x}^{t}(t-u) g^{\prime \prime}(u) d u ; x\right) \\
\leq & g(x)+\tilde{M}_{n, p_{n}, q_{n}}\left(\int_{x}^{t}(t-u) g^{\prime \prime}(u) d u ; x\right) \\
- & \int_{x}^{\frac{1}{[2] p_{n}, q_{n} q_{n}}}\left(2 x+\frac{\left(p_{n}^{n-1}-q_{n}^{n}-1, x\right)}{q_{n}[n-1] p_{n}, q_{n}}\right) \\
& \left(\frac{1}{[2]_{p_{n}, q_{n}} q_{n}}\left(2 x+\frac{\left(p_{n}^{n-1}-q_{n}^{n-1} x\right)}{q_{n}[n-1]_{p_{n}, q_{n}}}\right)-u\right) g^{\prime \prime}(u) d u .
\end{aligned}
$$

Further, we have

$$
\begin{aligned}
& \left|M_{n, p_{n}, q_{n}}(g(t) ; x)-g(x)\right| \leq \tilde{M}_{n, p_{n}, q_{n}}\left(\left|\int_{x}^{t}(t-u) g^{\prime \prime}(u) d u\right| ; x\right) \\
& +\left|\int_{x}^{\frac{1}{[2] p_{n}, q_{n} q_{n}}}\left(2 x+\frac{\left(p_{n}^{n-1}-q_{n}^{n-1} x\right)}{q_{n}[n-1] p_{n}, q_{n}}\right)\right| \frac{1}{[2]_{p_{n}, q_{n}} q_{n}}\left(2 x+\frac{\left(p_{n}^{n-1}-q_{n}^{n-1} x\right)}{q_{n}[n-1]_{p_{n}, q_{n}}}\right)-u|| g^{\prime \prime}(u)|d u| \\
& \leq \tilde{M}_{n, p_{n}, q_{n}}\left((t-x)^{2} ; x\right)\left\|g^{\prime \prime}(x)\right\|+\left(\frac{1}{[2]_{p_{n}, q_{n}} q_{n}}\left(2 x+\frac{\left(p_{n}^{n-1}-q_{n}^{n-1} x\right)}{q_{n}[n-1]_{p_{n}, q_{n}}}\right)-x\right)^{2}\left\|g^{\prime \prime}(x)\right\| \\
& =\delta_{n}^{2}(x)\left\|g^{\prime \prime}\right\| .
\end{aligned}
$$

Now, by boundedness of $\tilde{M}_{n, p_{n}, q_{n}}$, we get

$$
\begin{aligned}
\left|M_{n, p_{n}, q_{n}}(f ; x)\right| & \leq\left|\tilde{M}_{n, p_{n}, q_{n}}(f ; x)\right|+|f(x)| \\
& +\left|f\left(\frac{1}{[2]_{p_{n}, q_{n}} q_{n}}\left(2 x+\frac{\left(p_{n}^{n-1}-q_{n}^{n-1} x\right)}{q_{n}[n-1]_{p_{n}, q_{n}}}\right)\right)\right|, \\
& \leq 3\|f\| .
\end{aligned}
$$


Finally, we obtain

$$
\begin{aligned}
& \left|\tilde{M}_{n, p_{n}, q_{n}}(f ; x)-f(x)\right| \\
& =\left|M_{n, p_{n}, q_{n}}(f ; x)-f(x)+f\left(\frac{1}{[2]_{p_{n}, q_{n}} q_{n}}\left(2 x+\frac{\left(p_{n}^{n-1}-q_{n}^{n-1} x\right)}{q_{n}[n-1]_{p_{n}, q_{n}}}\right)\right)-f(x)\right| \\
& \leq\left|M_{n, p_{n}, q_{n}}(f-g ; x)\right|+\left|M_{n, p_{n}, q_{n}}(g ; x)-g(x)\right|+|g(x)-f(x)| \\
& +\left|f\left(\frac{1}{[2]_{p_{n}, q_{n}} q_{n}}\left(2 x+\frac{\left(p_{n}^{n-1}-q_{n}^{n-1} x\right)}{q_{n}[n-1]_{p_{n}, q_{n}}}\right)\right)-f(x)\right| \\
& \leq 4\|f-g\|+\delta_{n}^{2}(x)\left\|g^{\prime \prime}(x)\right\| \\
& +\omega\left(f,\left|\frac{1}{[2]_{p_{n}, q_{n}} q_{n}}\left(\left(2-[2]_{p_{n}, q_{n}} q_{n}\right) x+\frac{\left(p_{n}^{n-1}-q_{n}^{n-1} x\right)}{q_{n}[n-1]_{p_{n}, q_{n}}}\right)\right|\right) .
\end{aligned}
$$

By taking the infimum on the right hand side over all $g \in \mathbb{W}^{2}$, we get

$$
\left|\tilde{M}_{n, p_{n}, q_{n}}(f ; x)-f(x)\right| \leq 4 K_{2}\left(f, \delta_{n}^{2}(x)\right)+\omega\left(f, \alpha_{n}(x)\right) .
$$

Finally, by using the property of $K$-functional, we obtain

$$
\left|\tilde{M}_{n, p_{n}, q_{n}}(f ; x)-f(x)\right| \leq C \omega_{2}\left(f, \delta_{n}(x)\right)+\omega\left(f, \alpha_{n}(x)\right) .
$$

Hence the proof is completed.

\section{GRAPHICAL EXAMPLES}

In this section, we estimate approximation for functions $f(x)=\sin (x)$ (Figure (1)), $f(x)=$ $(x-4 / 5)(x-2 / 3)(x-1 / 4)$ (Figure (2)), $f(x)=(x-2 / 3)(x-1 / 4)$ (Figure (3)) and $f(x)=\exp (x)$ (Figure (4)), by $(p, q)$-Meyer-König-Zeller Kantrovich operators using Matlab programming.

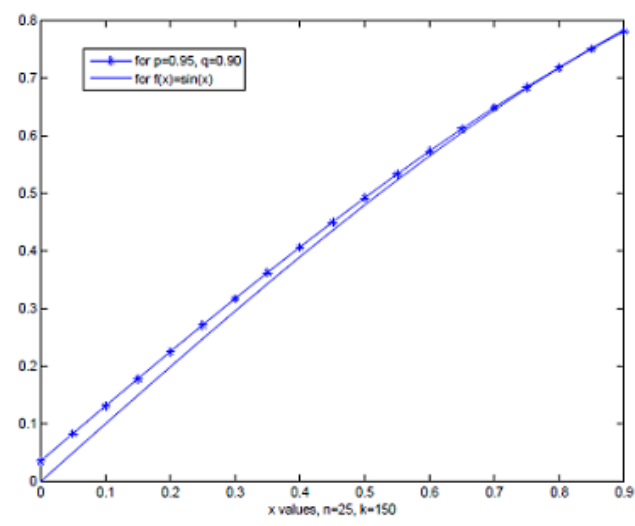

FIGURE 1. $f(x)=\sin (x)$ 


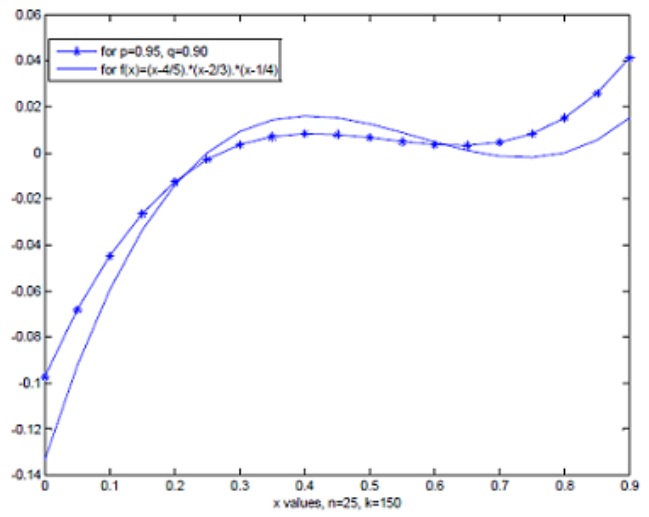

FIGURE 2. $f(x)=(x-4 / 5)(x-2 / 3)(x-1 / 4)$

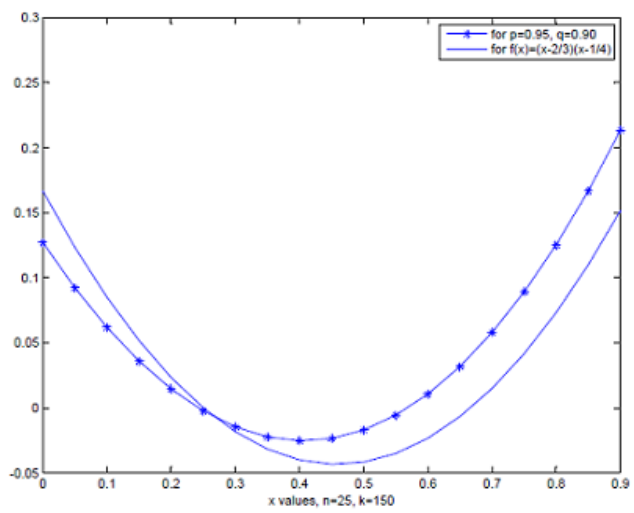

FIGURE 3. $f(x)=(x-2 / 3)(x-1 / 4)$

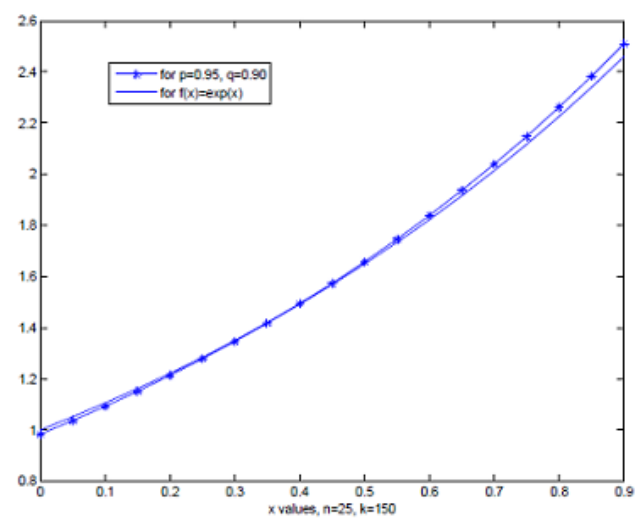

FIGURE 4. $f(x)=\exp (x)$ 


\section{REFERENCES}

[1] T. Acar: $(p, q)$-Genralization of Sźasz-Mirakyan operators. Math. Methods Appl. Sci., 39(10) (2016), 2685-2695.

[2] R. Chakrabarti and R. Jagannathan: $A(p, q)$-oscillator realization of two parameter quantum algebras. J. Phys. A: Math. Gen., 24 (1991), 711-718.

[3] E. W. Cheney and A. Sharma: Bernstein power series. Canad. J. Math., 16 (1964),241-252.

[4] R. A. DeVore and G. G. Lorentz. Construtive Approximation, Springer, Berlin, (1993).

[5] O. Dŏgru and O. Duman: Statistical approximationof Meyer-König and Zeller operators based on q-integers. Publ. Math. Debrecen, 68(1-2) (2006), 199-214.

[6] O. Dŏgru, O. Duman and C. Orhan: Statistical approximation by generalized Meyer-König and Zeller type operators., Studia Sci. Math. Hungar, 40(3) (2003), 359-371.

[7] O. Dŏgru and V. Gupta: Kovrokin-type approximation properties of bivariate q-Meyer-König and Zeller operators. Calcolo, 43(1) (2006), 51-63.

[8] V. Gupta and A. Aral: Bernstein Durmeyer operators based on Two Parameters. SER. Math. Inform, 31(1) (2016), 79-95.

[9] V. Gupta and H. Sharma: Statistical approximation by q-integrated Meyer-König-Zeller-Kantorovich operators. Craetive Math. Inf., 19(1) (2010), 45-52.

[10] V. Gupta, H. Sharma, T. Kim and S. H. Lee: Properties of q-analogue of Beta operator. Advances in Difference Equations, 2012:86 (2012).

[11] U. Kadak, A. Khan and M. Mursaleen: Approximation by Meyer-König and Zeller Operators using $(p, q)-$ Calculus. arXiv:1603.08539v2, (2016).

[12] K. Khan and D. K. Lobiyal: Bezier curves based on Lupas $(p, q)$ analogue of Bernstein polynomials in CAGD. arXiv: 1505.01810[cs.GR].

[13] P. P. Korovkin: Linear operators and approximation theory. Hindustan Publishing Corporation, Delhi, (1960).

[14] G. G. Lorentz: Berstein polynomials. Mathematical Expositions, University of Toronto Press: Toronto, 8 (1953).

[15] M. Mursaleen, K. J. Ansari and A. Khan: On $(p, q)$-analogue of Bernstein Operators. Appl. Math. Comput., 266 (2015), 874-882,(Erratum to: On $(p, q)$-analogue of Bernstein Operators, Appl. Math. Comput., 266 (2015), 874882.

[16] M. A. Ozarslan and O. Duman: Approximation theorems by Meyer-König and Zeller type operators. Chaos Solitons and Fractals.

[17] C. Radu: Statistical approximation by some linear operators of discrete type. MisKolc Math. Notes, 9(1) (2008), 61-68.

[18] P. N. Sadjang: On the fundamental theorem of $(p, q)$-calculus and some $(p, q)$-Taylor formulas. arXiv:1309.3934 [math.QA].

[19] H. Sharma and C. Gupta: On $(p, q)$-generalization of Szász-Mirakyan Kantorovich operators. Bollettino dell'Unione Matematica Italiana, 8(3) (2016), 213-222.

[20] H. Sharma: Note on approximation properties of generalized Durrmeyer operators. Mathematical Sciences, 6(1) (2012), $1-6$.

[21] T. Trif: Meyer-König and Zeller Operators based on the q-integers. Rev. Anal. Numer. Theor. Approx., 29(2) (2000), 221-229.

[22] W. Meyer-König and K. Zeller: Bernsteinsche Potenzreihen. studia math., 19 (1960), 89-94.

Gulzar Group of Institutes,

KhanNA, Ludhiana, PUnJab, India

E-mail address: pro.sharma.hegmail.com

Gulzar Group of Institutes,

KhanNA, Ludhiana, PUnJab, INDiA

E-mail address: ramapat imaurya@gmail.com

I. K. Gujral Punjab Technical University, DEPARTMENT OF MATHEMATICS

KAPURTHALA, PUNJAB, INDIA

E-mail address: guptacheena21@gmail.com 